\title{
Larval fish assemblages off central Chile upwelling ecosystem
}

\author{
Asociaciones ictioplanctónicas del ecosistema de surgencias de Chile central \\ Mauricio F. Landaeta ${ }^{1}$, Rodrigo Veas ${ }^{2,3}$, Jaime Letelier ${ }^{3}$ and Leonardo R. Castro $^{2,4}$ \\ ${ }^{1}$ Centro de Investigación en Nutrición, Tecnología en Alimentos y Sustentabilidad, CIEN Austral, Universidad Austral de Chile sede \\ Puerto Montt, Casilla 1327, Puerto Montt, Región de los Lagos, Chile \\ ${ }^{2}$ Laboratorio de Oceanografia Pesquera y Ecología Larval, Departamento de Oceanografía, Universidad de Concepción, \\ P.O. Box 160-C, Concepción, Chile \\ ${ }^{3}$ Doctorate Program in Oceanography, Universidad de Concepción, Chile \\ ${ }^{4}$ Center for Oceanographic Research in the Eastern South Pacific (COPAS), Universidad de Concepción, Chile \\ mlandaeta@cienaustral.cl
}

\begin{abstract}
Resumen.- Se detectaron diferencias espaciales (a escala vertical y horizontal) y estacionales en las asociaciones de larvas de peces recolectadas en estaciones fijas sobre el talud y plataforma continental frente a Chile central muestreadas por 24 h durante octubre de 1998 y julio de 1999. Durante octubre, la columna de agua en las estaciones de la plataforma y talud estuvo caracterizada por aguas frías y salinas, indicativa de un reciente evento de surgencia; durante este periodo las asociaciones de larvas de peces estuvieron estructuradas por la hora del día en la estación de la plataforma. Esta asociación larval estuvo dominada por el pez mesopelágico Maurolicus parvipinnis y anchoveta Engraulis ringens. Por otra parte, sobre el talud continental, el ictioplancton estuvo estructurado verticalmente, con diferentes asociaciones en función de la profundidad. La asociación estuvo dominada por M. parvipinnis y el mictófido Hygophum bruuni. Durante julio de 1999, las condiciones oceanográficas se caracterizaron por una mayor temperatura superficial, inversión térmica y una capa superficial de baja salinidad $(33,6-33,8)$. Las asociaciones ictioplanctónicas de la plataforma y el talud estuvieron estratificadas verticalmente, y se agregaron bajo la haloclina. Las larvas de E. ringens y $H$. bruuni fueron altamente abundantes en ambas estaciones, y varias especies de hábitat submareal/intermareal (Hypsoblennius sordidus, Sebastes capensis) fueron recolectadas sobre la plataforma. La importancia de las características oceanográficas en la estructura de las asociaciones de larvas de peces frente a Chile central cambió entre ambos periodos de muestreo, siendo más notable durante julio (efectos de la capa de baja salinidad) que en octubre (presencia de aguas de surgencia).
\end{abstract}

Palabras clave: Ictioplancton, Pacífico suroriental, surgencia, haloclina

\section{Introduction}

In eastern boundary systems worldwide, wind-driven coastal upwelling injects nutrients to the photic layer producing high levels of primary production and, as such, constitutes one of the most important mesoscale oceanic

\begin{abstract}
Spatial (both vertical and horizontal) and seasonal differences were detected in larval fish assemblages from samples collected off central Chile. Vertically-stratified sampling was carried out over the slope and continental shelf off Talcahuano in central Chile, during October 1998 and July 1999 to examine differences in larval fish assemblages. During October, the entire water column in the shelf and slope stations was characterized by cold and saltier waters, indicative of a recent upwelling event; during this period the larval fish assemblage was structured by time of the day in the shelf station. This assemblage was dominated by larvae of the mesopelagic lightfish Maurolicus parvipinnis and anchoveta Engraulis ringens. By contrast, ichthyoplankton was vertically structured in the slope station, showing different taxa associations in function of the depth (largely dominated by M. parvipinnis and the myctophid Hygophum bruuni). During July 1999, oceanographic conditions were characterized by higher surface temperatures, thermal inversion and a low salinity surface layer (33.6-33.8). Larval fish assemblages from slope and shelf were vertically stratified, and were aggregated below the halocline. Larval E. ringens and $H$. bruuni were highly abundant in both stations, and larvae of several taxa of subtidal/intertidal habitat (Hypsoblennius sordidus, Sebastes capensis) were collected over the shelf. During both periods the oceanographic features affecting the structure of larval fish assemblages off central Chile changed, wich was more noticeable during July (effects of the low salinity layer) than October (presence of recently upwelled waters).
\end{abstract}

Key words: Ichthyoplankton, southeast Pacific, upwelling, halocline

physical processes (Daneri et al. 2000, Montero et al. 2007). Along the southeast Pacific Ocean, the Humboldt Current System supports one of the largest epipelagic fisheries in the world (Bakun 1996). An important upwelling area is located off Talcahuano in central Chile $\left(36^{\circ} \mathrm{S}, 73^{\circ} \mathrm{W}\right)$, which is characterized by the seasonal 
occurrence of 3-10 d southerly and southwesterly wind events during spring-summer, producing alternating upwelling and relaxation conditions (Strub et al. 1998, Figueroa \& Moffat 2000, Sobarzo et al. 2007). The wind forcing generates upwelling of cold, nutrient-rich Equatorial SubSurface Waters (ESSW) leading to subsequent increases in primary and secondary production rates (Daneri et al. 2000) and therefore, favourable feeding conditions for larval fishes among other plankton organisms (i.e. high concentrations of copepod eggs and nauplii; Grunewald et al. 2002). However, if southerly wind events are frequent and/or persistent, the probability of offshore larval transport through the Ekman surface layer increases. Conversely, the prevailing northerly winds during late autumn and winter induce a net coastward flux of the Ekman layer, enabling the maintenance of less cold Surface SubAntartic Waters (SSAW) which favours the retention of fish eggs and larvae along the coast, although productivity is substantially lower than during the upwelling season (Castro et al. 2000, Cubillos et al. 2007, Montero et al. 2007). Also, others processes at seasonal scale may influence the vertical structure of the water column, such as the mixed layer's heat balance which is dominated by solar radiation during summer, and the freshwater balance, which is dominated by river discharge and precipitation with maxima in June and July (Sobarzo et al. 2007). Under this scenario, marine fish populations living in the area must adjust their reproductive tactics to deal with environmental heterogeneity and thereby maximize larval survival (Castro et al. 2000, Landaeta \& Castro 2002, Sponaugle et al. 2002, Landaeta et al. 2006, Lett et al. 2007).

Life histories of fish species may evolve to synchronize their reproductive activities to the frequency of certain oceanographic events (Bakun 1996, Sponaugle et al. 2002) which implies spawning at selected areas and times. The co-occurrence of individual species in a larval fish assemblage not only results from a similar spawning behaviour of several species, but it also suggests that they share common requirements during their early life history (Nonaka et al. 2000). An approach for understanding the sympatric occurrence of eggs and larvae of marine fishes and the influence of physical and biological processes on these associations is to study the structure and dynamic of larval fish assemblages. (Smith et al. 1999, Berasategui et al. 2004, Espinosa-Fuentes \& Flores-Coto 2004, Vélez et al. 2005, Peguero-Icaza et al. 2008). For example, Sánchez-Velasco et al. (2006) described that cyclonic circulation inside a shallow bay was linked to the spatial distribution of a coastal assemblage that spread out in the same area of circulation. Presence of more stable water column, wind induced mixing and the depth and the strength of the thermocline may also influence the abundance and vertical distribution of larval fish assemblages (Gray \& Kingsford 2003, Sabatés 2004, Sánchez-Velasco et al. 2007). Also, even in very nearshore areas, larval fish assemblages seem to be vertically structured at a small scale (a few meters) (Borges et al. 2007a).

Despite the importance of the coasts of southeast Pacific, particularly central-south Chile, as some of the most productive fishing areas in the world, larval fish assemblage studies are scarce. These studies have considered interannual (Loeb \& Rojas 1988) and seasonal variability (Balbontín \& Bravo 1999, Hernández-Miranda et al. 2003, Vélez et al. 2005) and the effects of El Niño events (Rodriguez-Graña \& Castro 2003), but all of them off northern and central Chile. However, there are several aspects about spatial and temporal variability of larval fishes off central-south Chile which are still unknown, and to date there is no information on the vertical structure of larval fish assemblages in this zone. Since this area is affected by environmental variability in a seasonal frequency as upwelling events during spring-summer, and drastic increases of the freshwater input in the coastal area during winter, we should also expect seasonal differences of the fish larval assemblages structure due to physical forcing (advection, vertical structure of the water column) during upwelling favourable (October) and non favourable (July) months over the continental shelf and slope. Therefore, our hypothesis is that spatial (shelf and slope) and temporal (October-July) variability of larval fish assemblages off central Chile is related with seasonal occurring mesoscale physical processes (e.g. upwelling).

\section{Material and methods}

\section{Study area}

The study area $\left(36-37^{\circ} \mathrm{S}\right.$, Fig. 1) is located at the coastal edge of the northward-flowing Humboldt Current system off central Chile, and is bounded by the Itata Canyon to the north and the Biobio Canyon to the south. The continental shelf narrows from $60 \mathrm{~km}$ in the north to 25 $\mathrm{km}$ in the south over an alongshore expanse of $70 \mathrm{~km}$ (Sobarzo \& Djurfeldt 2004). On this shelf, there are several embayments utilized as spawning and nursery zone by several marine fishes (Castillo et al. 1991, Landaeta \& Castro 2006a), two important rivers (Biobio and Itata), and one prominent point (Punta Lavapié) that is an important coastal upwelling site in central Chile.

\section{Sampling design and collection of samples}

Oceanographic cruises were carried out during 18-23 October 1998 (MIRC I, spring) and 9-14 July 1999 (MIRC II, winter) onboard RV Abate Molina, as part of a 


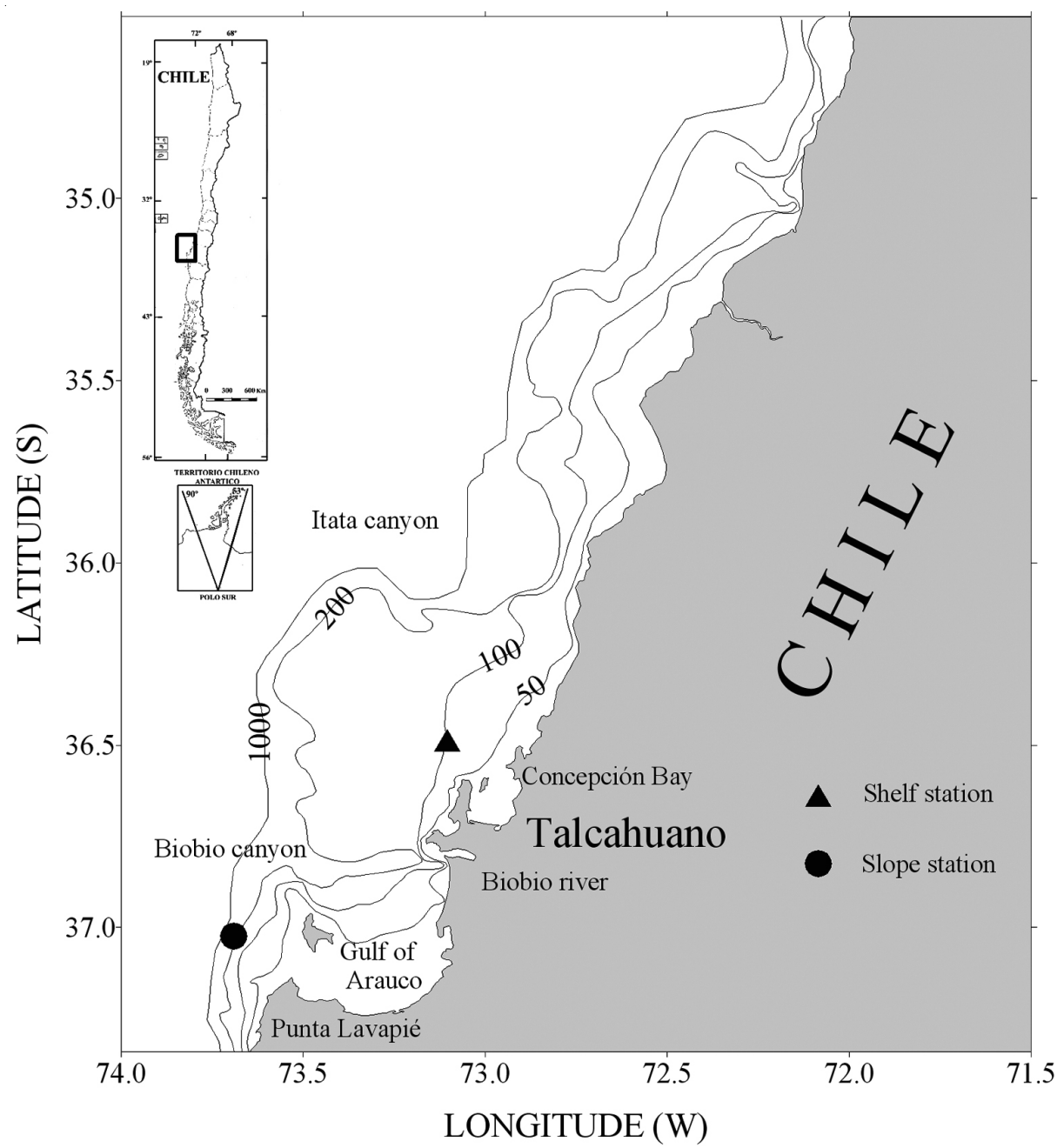

Figure 1

Map showing the location of stations where 24-h sampling was carried out over the continental shelf (black triangle) and slope (black circle) in central Chile during October 1998 and July 1999

Mapa que muestra la ubicación de las estaciones de 24 h llevadas a cabo durante octubre de 1998 (primavera austral) y julio de 1999 (invierno austral), sobre la plataforma continental (triángulo negro) y talud (círculo negro) en Chile central

project designed to determine carbon flux rates in the Humboldt Current System (FONDAP-Humboldt) (Morales et al. 2007). During both cruises, two stations were sampled over a period of $24 \mathrm{~h}$, one over the continental shelf off Talcahuano and another on the slope off Punta Lavapié, Chile (Fig. 1). At these stations hydrographic casts were performed to $200 \mathrm{~m}$ or $10 \mathrm{~m}$ above the seafloor with a Seabird SB-19 CTD (Conductivity-Temperature-Depth) profiler. Other physical data obtained for the study period included selected satellite wind, sea surface temperatures (SST) and altimetry data. Wind stress data from the close to shore pixel $\left(73.5^{\circ} \mathrm{W}-36.5^{\circ} \mathrm{S}\right)$ was extracted from ERS 2 gridded images (spatial resolution of $111 \mathrm{~km}$ ) between 1998 and 2000. Images of Sea Surface Temperature (spatial resolution of $1.1 \mathrm{~km}$ ) were produced by the AVHRR sensor onboard NOAA satellite series and they correspond for 20 October 1998 and 13 July 1999. The geostrophic velocity field (spatial resolution of $27.75 \mathrm{~km}$ ) was obtained from the AVISO program (www.jason.oceanobs.com), as 10 days composite. Alongshore wind stress component estimates were computed from ERS wind reports according to the following bulk aerodynamics equation:

$\tau \quad=\quad \rho \quad C \quad{ }_{d}|V| V$ 
Table 1

Summary of the main characteristics of the surveys carried out

Resumen de las principales características de los cruceros realizados

\begin{tabular}{lcllc}
\hline Cruise & Survey dates & Station & \multicolumn{1}{c}{$\begin{array}{c}\text { Sampling strata } \\
(\mathrm{m})\end{array}$} & $\begin{array}{c}\text { Number of samples } \\
(\text { day/night })\end{array}$ \\
\hline MIRC I & $18-23$ October 1998 & Shelf & $0-25 ; 25-50$ & $12(6 / 6)$ \\
& & Slope & $0-50 ; 50-100 ; 100-150$ & $18(9 / 9)$ \\
MIRC II & $9-14$ July 1999 & Shelf & $0-10 ; 10-25 ; 25-50 ; 50-75$ & $24(12 / 12)$ \\
& & Slope & $0-25 ; 25-50 ; 50-100 ; 100-150$ & $20(12 / 8)$ \\
\hline
\end{tabular}

where $\tau$ is the stress vector, $\rho$ is the density of air, $V$ is the wind velocity, $|\mathrm{V}|$ is the wind speed and $\mathrm{C}_{\mathrm{d}}$ is a dimensionless drag coefficient (Okubo 1980).

Five to six oblique plankton tows (three during daytime and two or three at night) were carried out every 4 to $6 \mathrm{~h}$ during a period of $24 \mathrm{~h}$ at each station with an opening-closing $1-\mathrm{m}^{2}$ mouth Tucker trawl, equipped with three $250-\mu \mathrm{m}$ mesh nets and a General Oceanic flowmeter mounted on the frame. The net was obliquely deployed to a maximum depth of $150 \mathrm{~m}$ or near the seabed in depths $<150 \mathrm{~m}$, and two to four strata were sampled (Table 1) depending on bottom depth. Towing speed was c. 2-3 knots. Volume filtered in each tow (October 1998: range $=61-1306 \mathrm{~m}^{3}$ tow $^{-1}$, mean $=423 \mathrm{~m}^{3}$ tow $^{-1}$, July 1999: range $=64-536 \mathrm{~m}^{3}$ tow $^{-1}$, mean $=313 \mathrm{~m}^{3}$ tow $^{-1}$ ) was calculated from flowmeter counts. After recovery, the nets were rinsed and ichthyoplankton samples preserved in $4 \%$ buffered formaldehyde.

All larval fishes were removed from samples, counted and identified to the lowest possible taxonomic level using descriptions by Fahay (1983), Moser (1996), and Neira et al. (1998). Larvae were classified according to the adult habitat as mesopelagic, epipelagic, demersal or subtidal/ intertidal, and the concentration of each taxon (larvae $1000 \mathrm{~m}^{-3}$ ) estimated at each stratum for comparisons in the vertical axis. The integrated abundance of larvae in the water column (larvae $10 \mathrm{~m}^{-2}$ ) was also estimated for each sampling station to compare the ichthyoplankton composition between shelf and slope irrespective of the sampled strata.

\section{Data analyses}

Multivariate analyses were performed to determine the seasonal and vertical variability of larval fish assemblages utilizing the software package Primer 5. Standardized ichthyoplankton abundance integrated in sampled the water column (larvae $10 \mathrm{~m}^{-2}$ ) was fourth root transformed to enhance the contribution of less abundant taxa and a Bray-Curtis similarity matrix was generated from these data. Similarities between assemblages were graphically represented by non-metric multidimensional scaling (MDS, Cox \& Cox 2000) ordination. Goodness of fit was determined by a stress coefficient. Classification and MDS ordination were performed using abundance of larval fishes by strata (larvae $1000 \mathrm{~m}^{-3}$ ) instead of integrated values to determine vertical structure of ichthyoplankton assemblages by sampling month (October and July) and location (shelf and slope).

\section{Results}

\section{Oceanographic conditions}

Persistent alongshore south winds during October 1998 favoured upwelling events and produced negative (offshore) Ekman transport (mean $\pm 1 \mathrm{SD},-4.40 \pm 0.34$ $\mathrm{m}^{2} \mathrm{~s}^{-1}$ ) (Fig. 2). Sea surface temperature (SST) showed horizontal heterogeneity, with areas of colder, recently upwelled waters $\left(<10^{\circ} \mathrm{C}\right)$ in nearshore waters off Punta Lavapié and Concepción Bay (Fig. 3a). The larger upwelling plume of cold water $\left(12.5-13^{\circ} \mathrm{C}\right)$ originated off Punta Lavapié was advected offshore and extended westward to $75^{\circ} \mathrm{W}$ as an anticyclone filament. The latter structure coincides spatially with an anticyclone vortex observed through geostrophic current vectors (Fig 3). Altimetry derived speeds were higher $\left(>30 \mathrm{~cm} \mathrm{~s}^{-1}\right)$ than surrounding waters, particularly between the coastal zone and the filament. The CTD-derived data over the slope and continental shelf showed almost identical surface $(<5$ m) temperatures ranging between $11.29 \pm 0.37^{\circ} \mathrm{C}$ and $11.30 \pm 0.22^{\circ} \mathrm{C}$, respectively (Fig. 4). However, mean surface salinity at the slope station was lower (34.05 \pm $0.05)$ than that at the shelf station $(34.32 \pm 0.04)$. In deeper waters, there was a weak thermocline at both stations, varying between 25 and $49 \mathrm{~m}$ depth over the shelf, and between 40 and $60 \mathrm{~m}$ in the slope. In the slope station a surface thermocline was observed between 5 and $15 \mathrm{~m}$ deep. With the increase of temperature there was also a decrease in salinity. A pycnocline was observed at both stations, but was more intense over the shelf. 


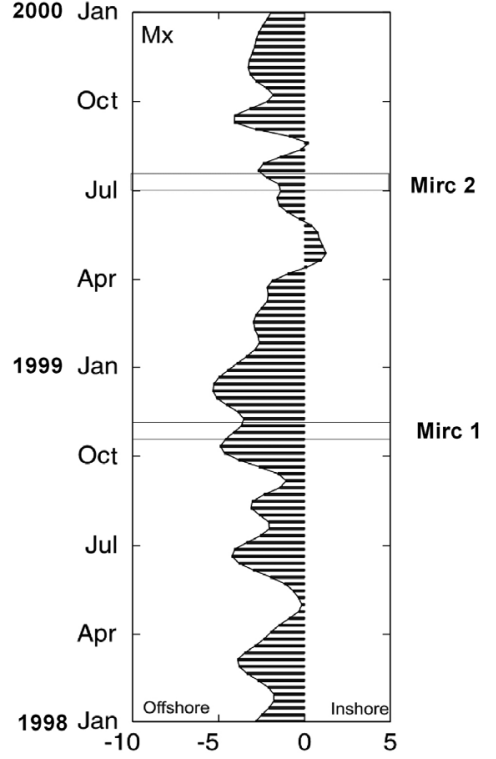

Figure 2

Time series of Ekman transport $\left(\mathrm{m}^{2} \mathrm{~s}^{-1}\right)$ off Talcahuano area during 1998 and 1999. Mirc 1 and Mirc 2 correspond to sampling dates

Serie de tiempo de transporte de Ekman $\left(\mathrm{m}^{2} \mathrm{~s}^{-1}\right)$ frente a Talcahuano durante 1998 y 1999. Mir 1 y Mirc 2 corresponden a las fechas de muestreo

The wind pattern during July 1999 was dominated by weaker, highly variable equatorward wind stress, and lower offshore Ekman transport $\left(-1.84 \pm 0.49 \mathrm{~m}^{2} \mathrm{~s}^{-1}\right)$ than during spring (Fig. 2). The SST images showed an irregular spatial pattern, and areas of cold waters and filaments were absent (Fig. 3b). In the coastal zone, especially in the Gulf of Arauco zone $\left(37^{\circ} \mathrm{S}\right)$, the predominant SST was around $12^{\circ} \mathrm{C}$. An irregular pattern of geostrophic velocity field resulted from altimetry data. However, a poleward flow following the bathymetry of the slope was evident (Fig. 3b), with increasing speeds $\left(\sim 20 \mathrm{~cm} \mathrm{~s}^{-1}\right)$ off Punta Lavapié. There was a clear thermal inversion along the mixed layer at the slope station, with a subsurface maximum temperature of $13.05 \pm 0.22^{\circ} \mathrm{C}$ at a depth of $35 \mathrm{~m}$ (Fig. 4). This thermal inversion was associated with a surface low salinity layer at $35 \mathrm{~m}$ which ranged from $33.71 \pm 0.05$ to $34.12 \pm 0.04$. Below the low-salinity layer, temperature values decreased and salinity increased steeply. At the shelf station, the mixed layer was deeper than that in the slope $(\sim 50 \mathrm{~m})$ and uniform in temperature, but the salinity increased with depth from $33.85 \pm 0.07$ to $34.09 \pm 0.09$. Density profiles indicated a stable water column in the first $30 \mathrm{~m}$ depth with a pycnocline at $50 \mathrm{~m}$ (Fig. 4).

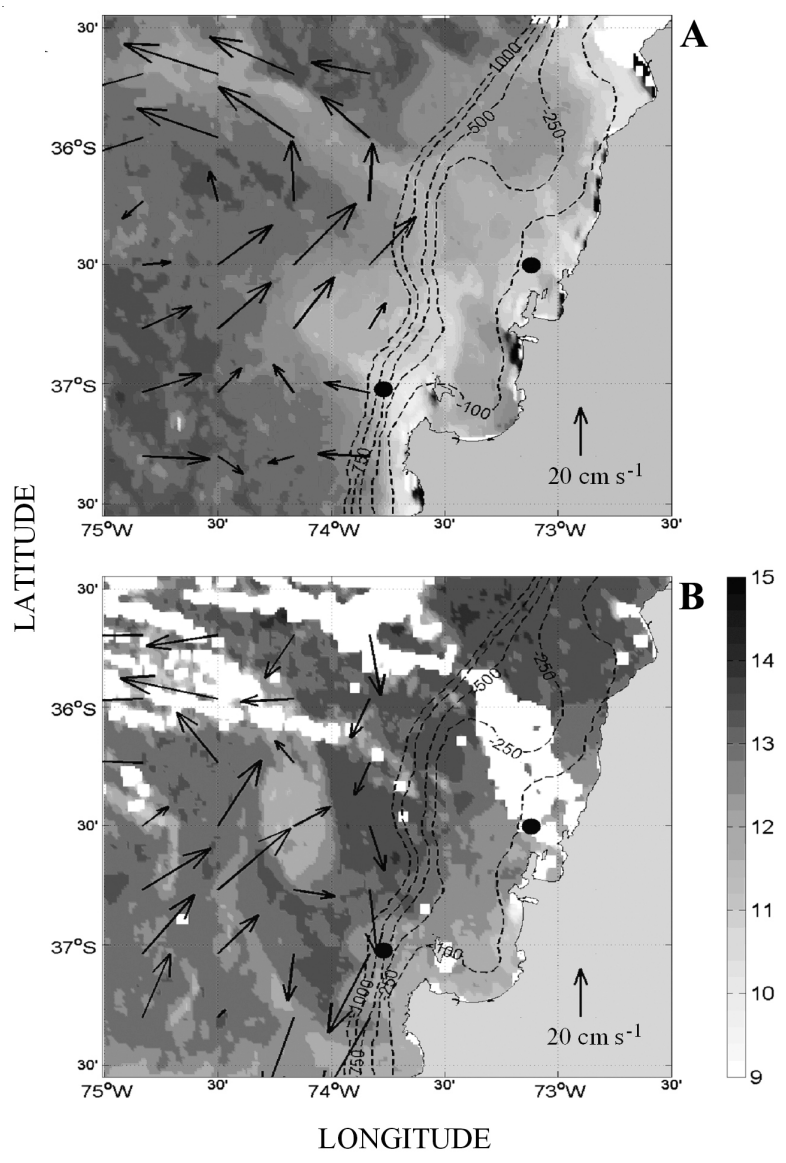

Figure 3

Sea surface temperature $\left({ }^{\circ} \mathrm{C}\right)$ image and geostrophic flows $\left(\mathrm{cm} \mathrm{s}^{-1}\right)$ off central Chile during October 201998 (a) and July 131999 (b). Image taken from AVHRR sensor

Temperatura Superficial del Mar $\left({ }^{\circ} \mathrm{C}\right)$ y flujos geostróficos $\left(\mathrm{cm} \mathrm{s}^{-1}\right)$ frente a Chile central durante el 20 de octubre de 1998 (a) y el 13 de julio de 1999 (b). Imagen tomada por el sensor AVHRR

\section{Larval fish assemblages}

A total of 10,961 larval fishes were captured during both cruises. These comprised 34 taxa representing 25 families (Table 2). The MDS ordination plots of similarities for integrated data defined four assemblages according to sampling month (October and July) and location (shelf and slope): an October Shelf Assemblage (OSH), October Slope Assemblage (OSL), July Shelf Assemblage (JSH), and a July Slope Assemblage (JSL) (Fig. 5).

The OSH assemblage was dominated by larval anchoveta Engraulis ringens $\left(\right.$ mean $=23$ larvae $\left.10 \mathrm{~m}^{-2}\right)$, common sardine Strangomera bentincki (21 larvae 10 $\mathrm{m}^{-2}$ ) and mesopelagic lightfish Maurolicus parvipinnis (24 larvae $10 \mathrm{~m}^{-2}$ ) (Table 2). Other species such as hake 


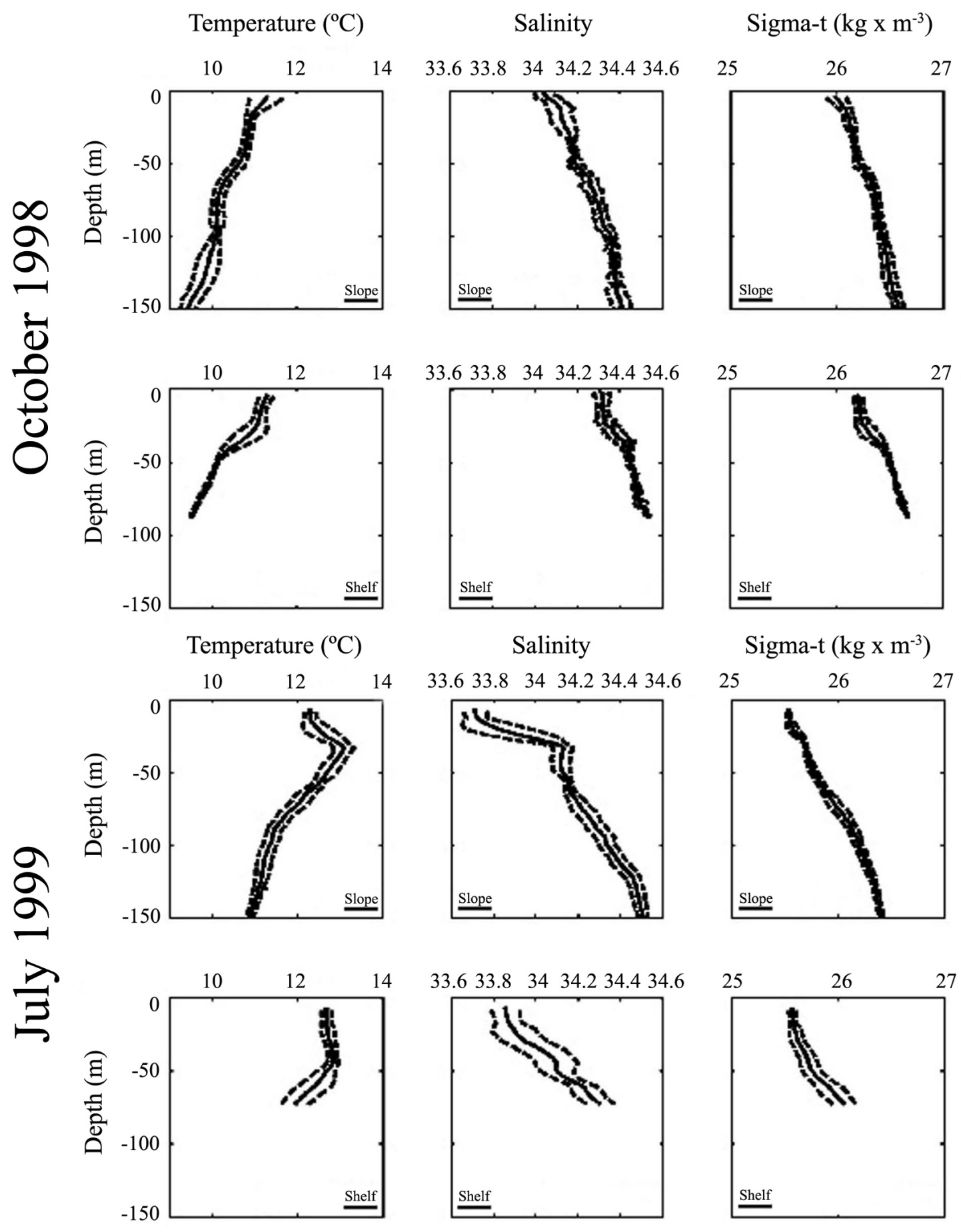

Figure 4

CTD derived water temperature $\left({ }^{\circ} \mathrm{C}\right)$, salinity and sigma-t $\left(\mathrm{kg} \mathrm{x} \mathrm{m}^{-3}\right)$ during Mirc 1 (October 1998) and Mirc 2 (July 1999) surveys over continental slope and shelf

Perfiles verticales de temperatura $\left({ }^{\circ} \mathrm{C}\right)$, salinidad y sigma-t $\left(\mathrm{kg} \mathrm{x} \mathrm{m}^{-3}\right)$ derivados del CTD durante los cruceros Mirc 1 (octubre de 1998) y Mirc2 (julio de 1999) sobre la plataforma y talud continental 
Table 2

Composition of larval fishes collected during MIRC 1 (October 1998) and MIRC 2 (July 1999) cruises off Talcahuano area, central Chile. Larval abundances are expressed as individuals $10 \mathbf{~ m}^{-2}$; $\mathrm{SD}=$ standard deviation, $\% \mathrm{~F}=$ frequency of occurrence

Composición de larvas de peces recolectadas durante los cruceros MIRC 1 (octubre 1998) y MIRC 2 (julio 1999) frente al área de Talcahuano, Chile central. Las abundancias larvales están expresadas como individuos $10 \mathrm{~m}^{-2} ; \mathrm{SD}=$ desviación estándar, $\% \mathrm{~F}=$ frecuencia de ocurrencia

\begin{tabular}{|c|c|c|c|c|c|c|c|c|c|c|c|c|}
\hline & \multicolumn{6}{|c|}{ October 1998} & \multicolumn{6}{|c|}{ July 1999} \\
\hline & \multicolumn{3}{|c|}{ Continental Shelf } & \multicolumn{3}{|c|}{ Slope } & \multicolumn{3}{|c|}{ Continental Shelf } & \multicolumn{3}{|c|}{ Slope } \\
\hline & $\begin{array}{l}\text { Mean abundance } \\
\text { (ind per } 10 \mathrm{~m}^{2} \text { ) }\end{array}$ & SD & $\% \mathrm{~F}$ & $\begin{array}{l}\text { Mean abundance } \\
\text { (ind per } 10 \mathrm{~m}^{2} \text { ) }\end{array}$ & SD & $\% \mathrm{~F}$ & $\begin{array}{l}\text { Mean abundance } \\
\text { (ind per } 10 \mathrm{~m}^{2} \text { ) }\end{array}$ & SD & $\% \mathrm{~F}$ & $\begin{array}{l}\text { Mean abundance } \\
\text { (ind per } 10 \mathrm{~m}^{2} \text { ) }\end{array}$ & SD & $\% \mathrm{~F}$ \\
\hline \multicolumn{13}{|l|}{ Mesopelagic taxa } \\
\hline Bathylagus sp. & - & - & - & 1,65 & 0,91 & 0,07 & - & - & - & 0,94 & - & 0,11 \\
\hline Diogenichthys laternatus & - & - & - & - & - & - & 0,90 & 0,07 & 0,07 & 1,61 & 0,86 & 0,77 \\
\hline Hygophum bruuni & 1,16 & 0,35 & 0,98 & 30,28 & 23,58 & 3,89 & 102,36 & 53,53 & 15,61 & 148,36 & 183,97 & 88,77 \\
\hline Lampanyctodes hectoris & 2,11 & 1,52 & 1,78 & 30,00 & 14,51 & 3,85 & - & - & - & - & - & - \\
\hline Lampanyctus iselinoides & - & - & - & 3,33 & 0,47 & 0,14 & 1,61 & 1,26 & 0,12 & 4,51 & 6,12 & 2,16 \\
\hline Maurolicus parvipinnis & 24,28 & 20,44 & 25,62 & 546,79 & 397,46 & 70,24 & - & - & - & - & - & - \\
\hline Melamphaidae & - & - & - & 0,77 & - & 0,02 & - & - & - & - & - & - \\
\hline Protomyctophum chilensis & - & - & - & 14,01 & 18,44 & 1,80 & 0,64 & 0,25 & 0,03 & 1,02 & - & 0,12 \\
\hline Protomyctophum crockeri & - & - & - & 12,28 & - & 0,26 & - & - & - & 1,42 & 0,57 & 0,34 \\
\hline Engraulis ringens & 22,99 & 9,36 & 29,12 & 65,06 & 50,66 & 8,36 & 451,32 & 115,56 & 68,85 & 5,11 & 2,98 & 1,22 \\
\hline Ethmidium maculatum & - & - & - & 5,17 & 3,33 & 0,33 & 1,32 & - & 0,03 & - & - & - \\
\hline Normanichthys crockeri & 2,13 & 2,17 & 1,35 & 3,00 & - & 0,13 & 18,97 & 7,12 & 2,89 & - & - & - \\
\hline Sardinops sagax & - & - & - & 1,50 & - & 0,03 & - & - & - & - & - & - \\
\hline Strangomera bentincki & 20,52 & 22,24 & 25,99 & 41,40 & 19,76 & 4,43 & 5,75 & 3,47 & 0,88 & - & - & - \\
\hline Stromateus stellatus & - & - & - & - & - & - & 0,40 & - & 0,01 & - & - & - \\
\hline \multicolumn{13}{|l|}{ Demersal taxa } \\
\hline Coelorhynchus sp. & - & - & - & 2,44 & 1,42 & 0,21 & - & - & - & & & \\
\hline Genypterus sp. & - & - & - & 1,42 & 0,39 & 0,09 & 1,38 & - & 0,04 & 1,46 & 0,55 & 0,35 \\
\hline Hippoglossina macrops & 0,47 & - & 0,10 & - & - & - & 7,37 & 5,92 & 1,12 & 2,49 & 1,48 & 1,19 \\
\hline Merluccius gayi & 7,29 & 5,43 & 6,15 & 32,69 & 16,95 & 4,20 & 34,29 & 22,54 & 5,23 & 14,12 & 17,89 & 3,38 \\
\hline Moridae & - & - & - & 4,50 & - & 0,10 & - & - & - & - & - & - \\
\hline Ophichthyid sp. & 2,27 & - & 0,48 & 1,50 & - & 0,03 & - & - & - & - & - & - \\
\hline \multicolumn{13}{|l|}{ Subtidal and Intertidal taxa } \\
\hline Bovictus chilensis & - & - & - & - & - & - & 0,84 & 0,45 & 0,06 & - & - & - \\
\hline Congiopodus peruvianus & 0,47 & - & 0,10 & - & - & - & - & - & - & - & - & - \\
\hline Helcogrammoides chilensis & - & - & - & 1,83 & 1,04 & 0,12 & 0,72 & 0,57 & 0,06 & - & - & - \\
\hline Labrisomidae & - & - & - & - & - & - & 0,46 & - & 0,01 & - & - & - \\
\hline Leptonotus blainvilleanus & - & - & - & - & - & - & 2,34 & 1,59 & 0,24 & - & - & - \\
\hline Paralichthys microps & - & - & - & - & - & - & 1,32 & - & 0,03 & - & - & - \\
\hline Prolatilus jugularis & 0,92 & - & 0,19 & - & - & - & 3,38 & 1,54 & 0,52 & - & - & - \\
\hline Sciaenidae & - & - & - & - & - & - & 0,97 & - & 0,02 & - & - & - \\
\hline Sebastes capensis & 4,54 & 4,64 & 4,79 & 9,62 & 4,01 & 1,24 & 22,41 & 17,84 & 3,42 & 2,78 & 3,10 & 1,33 \\
\hline Syciases sanguineus & 1,42 & - & 0,30 & - & - & - & 0,77 & - & 0,02 & - & - & - \\
\hline
\end{tabular}




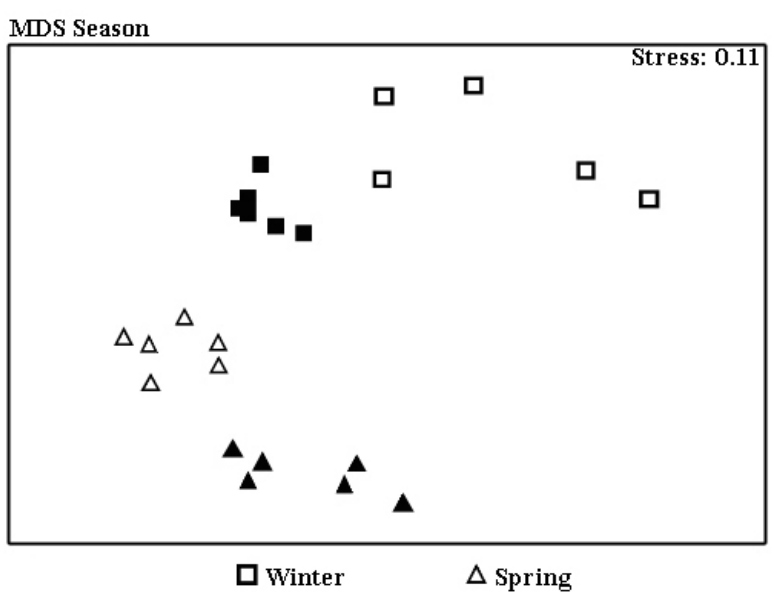

Figure 5

MDS plot showing larval fish assemblages for both surveys and locations. Black and white geometric figures correspond to samples collected in the slope and shelf stations, respectively

Gráfico de MDS mostrando las asociaciones de larvas de peces de ambos cruceros y localidades. Las figuras geométricas negra y blanca corresponden a muestras recolectadas en las estaciones del talud y plataforma, respectivamente
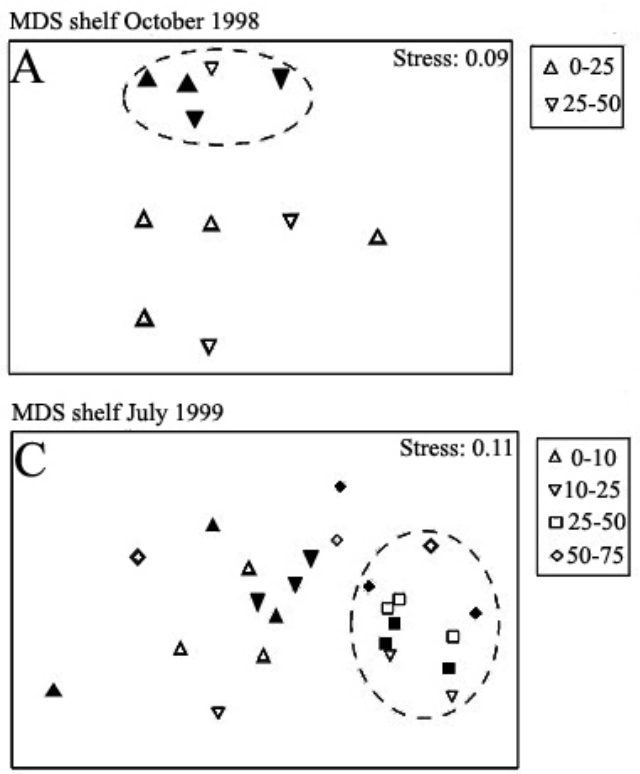

MDS slope October 1998

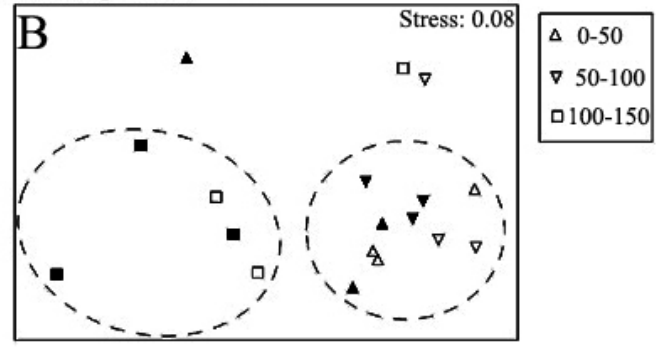

MDS slope July 1999

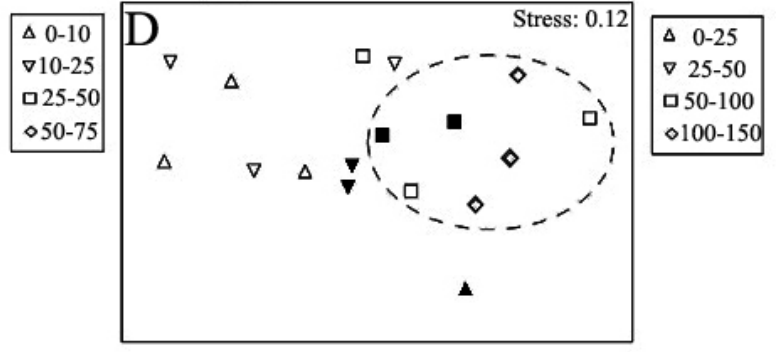

Figure 6

MDS plots showing vertical structure of larval fish assemblages for a) shelf October station, b) slope October station, c) shelf July station and d) slope July station. Black and white geometric figures correspond to night and day samples, respectively

Gráficos de MDS mostrando la estructura de las asociaciones de larvas de peces para a) estación de plataforma durante octubre, b) estación del talud durante Octubre, c) estación de plataforma durante julio, d) estación del talud durante julio. Las figuras geométricas negra y blanca corresponden a muestras de noche y día, respectivamente 


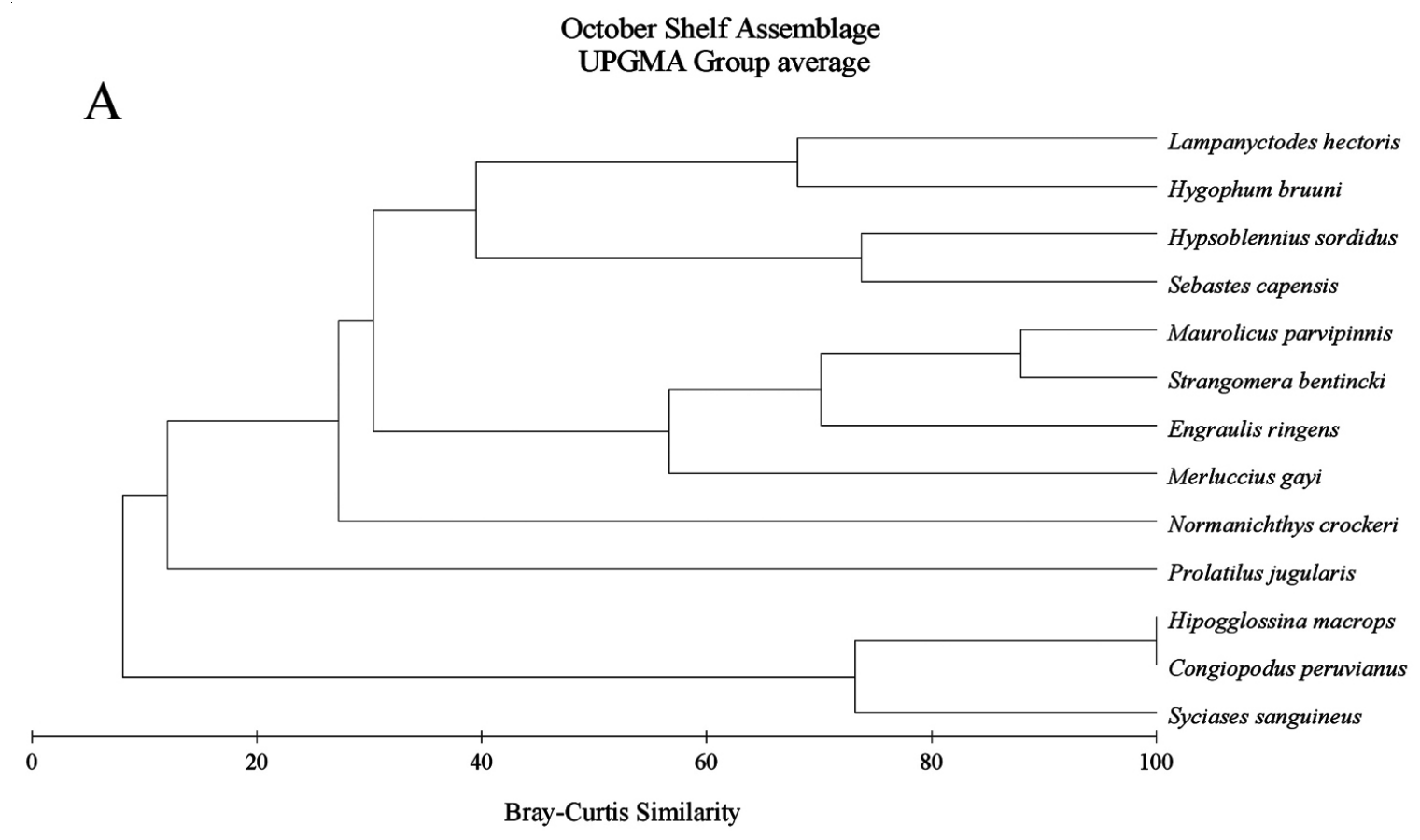

\section{B}

October Slope Assemblage

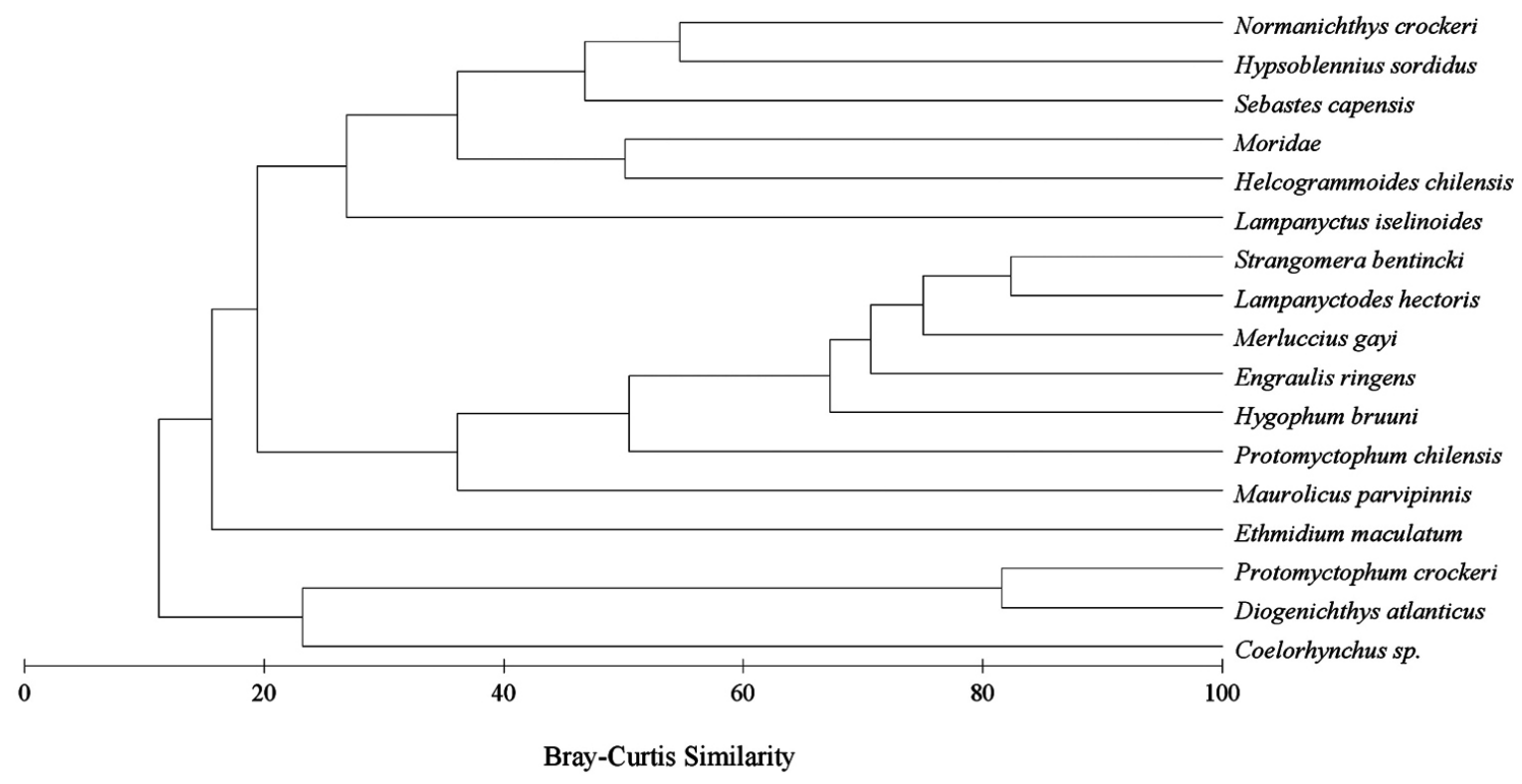

Figure 7

Cluster analysis showing relationship among larval fishes during October 1998 for the a) shelf station and b) slope station

Análisis de dendogramas mostrando la relación entre larvas de peces durante octubre de 1998 para la estación de a) plataforma y b) talud continental 
diverse over the slope (Table 2). Also, epipelagic larvae (13\%), and particularly larval clupeiforms (e.g. Engraulis, Strangomera) were more abundant in the slope than on the shelf during austral spring. The MDS plot showed that SSL was stratified according to depth, i.e. $>$ and < $100 \mathrm{~m}$ (Fig. 6b). The first group was formed by $N$. crockeri, H. sordidus, S. capensis, morids and Helcogrammoides chilensis (Fig. 7b) which were found exclusively $>100 \mathrm{~m}$, whereas the other taxa were collected throughout the water column (down to $150 \mathrm{~m}$ ), although in the deeper stratum (100-150 m) the larval abundances were considerably reduced (Table 3 ).

Larvae of epipelagic taxa dominated the JSH assemblage, with five taxa accounting for $73 \%$ of the total catch at the shelf station (Table 2). Most abundant species were anchoveta $\left(69 \% ; 450\right.$ larvae $\left.10 \mathrm{~m}^{-2}\right)$ and $N$. crockeri (19 larvae $10 \mathrm{~m}^{-2}$ ). Among the oceanic taxa, larval $H$. bruuni were the most abundant $\left(16 \% ; 106\right.$ larvae $\left.10 \mathrm{~m}^{-2}\right)$. Demersal fishes were represented by three species $(7 \%$ of total catch), whereas subtidal/intertidal fishes by 11

Table 3

Abundance at different depth strata of selected larval fishes captured during October 1998 off Talcahuano area, central Chile. Mean values of larval abundance (ind. $\left.1000 \mathrm{~m}^{-3}\right) \pm 1$ standard deviation

Abundancia a diferentes estratos de profundidad de algunas larvas de peces seleccionadas durante Octubre de 1998 frente al área de Talcahuano, Chile central. Valores promedios de abundancia larval (ind. $1000 \mathrm{~m}^{-3}$ ) \pm 1 desviación estándar

\begin{tabular}{|c|c|c|c|c|c|c|c|c|c|}
\hline \multirow[b]{3}{*}{ Taxa } & \multicolumn{4}{|c|}{ October Shelf Station } & \multicolumn{5}{|c|}{ October Slope Station } \\
\hline & \multicolumn{2}{|c|}{ Day } & \multicolumn{2}{|c|}{ Night } & \multirow[b]{2}{*}{$0-50$} & \multicolumn{2}{|l|}{ Day } & \multirow[b]{2}{*}{$0-50$} & \multirow{2}{*}{$\begin{array}{l}\text { Night } \\
50-100\end{array}$} \\
\hline & $0-25$ & $25-50$ & $0-25$ & $25-50$ & & $50-100$ & $100-150$ & & \\
\hline Engraulis ringens & $65.7 \pm 63.7$ & $37.1 \pm 21.4$ & $62.4 \pm 10.3$ & $26.5 \pm 27.1$ & $101.0 \pm 12.7$ & $83.5 \pm 113.8$ & 4,6 & $31.5 \pm 18.1$ & $69.5 \pm 85.3$ \\
\hline Strangomera bentincki & $12.7 \pm 7.4$ & $26.6 \pm 32.1$ & $124.6 \pm 36.5$ & $69.1 \pm 25.2$ & $26.5 \pm 3.5$ & $22.0 \pm 26.9$ & 7,6 & $25.3 \pm 7.1$ & $58.2 \pm 38.4$ \\
\hline Hygophum bruuni & 3,7 & 3,7 & $3.7 \pm 0.1$ & - & $17.6 \pm 15.1$ & $7.5 \pm 4.9$ & 13,6 & $43.6 \pm 33.6$ & $29.1 \pm 19.4$ \\
\hline Merluccius gayi & 5,9 & $17.9 \pm 19.7$ & $31.3 \pm 33.5$ & $6.1 \pm 3.4$ & $19.0 \pm 2.8$ & $42.0 \pm 18.4$ & $32.1 \pm 23.9$ & $16.2 \pm 16.1$ & $29.8 \pm 12.7$ \\
\hline Sebastes capensis & $16.4 \pm 1.5$ & 32,8 & 7,3 & 1,4 & $7.3 \pm 6.0$ & $21.0 \pm 9.9$ & - & $14.5 \pm 12.0$ & 3,0 \\
\hline Hypsoblennius sordidus & $14.7 \pm 10.2$ & $6.1 \pm 2.9$ & - & 1,4 & 12,0 & - & - & - & $9.0 \pm 4.2$ \\
\hline Maurolicus parvipinnis & $17.6 \pm 8.4$ & $42.9 \pm 55.1$ & $111.0 \pm 76.4$ & $62.4 \pm 10.5$ & $513.0 \pm 428.5$ & $751.5 \pm 352.8$ & $498.1 \pm 522.8$ & $211.0 \pm 141.2$ & $495.3 \pm 95.5$ \\
\hline Lampanyctodes hectoris & - & - & - & - & $21.2 \pm 13.7$ & 34,4 & 3,1 & $24.5 \pm 6.2$ & $47.4 \pm 12.7$ \\
\hline Protomyctophum chilensis & - & - & - & - & $26.4 \pm 27.6$ & $14.4 \pm 16.2$ & $15.2 \pm 19.3$ & 5,3 & $16.3 \pm 7.1$ \\
\hline
\end{tabular}

Table 4

Abundance at different depth strata of selected larval fishes captured during July 1999 off Talcahuano area, central Chile. Mean values of larval abundance (ind. $1000 \mathrm{~m}^{-3}$ ) \pm 1 standard deviation

Abundancia a diferentes estratos de profundidad de algunas larvas de peces seleccionadas durante Julio de 1999 frente al área de Talcahuano, Chile central. Valores promedios de abundancia larval (ind. $1000 \mathrm{~m}^{-3}$ ) \pm 1 desviación estándar

\begin{tabular}{|c|c|c|c|c|c|c|c|c|}
\hline \multirow{3}{*}{ Taxa } & \multicolumn{8}{|c|}{ July Shelf Station } \\
\hline & \multicolumn{4}{|c|}{ Day } & \multicolumn{4}{|c|}{ Night } \\
\hline & $0-10$ & $10-25$ & $25-50$ & $50-75$ & $0-10$ & $10-25$ & $25-50$ & $50-75$ \\
\hline Engraulis ringens & $91.9 \pm 49.4$ & $1004.0 \pm 1111.4$ & $784.8 \pm 307.9$ & $323.2 \pm 260.2$ & $143.6 \pm 121.6$ & $280.1 \pm 87.4$ & $1044.7 \pm 634.8$ & $593.1 \pm 420.9$ \\
\hline Hygophum bruuni & $32.8 \pm 30.9$ & $67.7 \pm 46.7$ & $240.5 \pm 222.9$ & $26.6 \pm 17.9$ & $19.1 \pm 14.7$ & $25.2 \pm 11.2$ & $247.5 \pm 83.8$ & $227.7 \pm 266.5$ \\
\hline Merluccius gayi & $14.1 \pm 16.8$ & $4.6 \pm 2.2$ & $71.8 \pm 19.9$ & $102.6 \pm 121.4$ & 31,3 & $5.4 \pm 0.9$ & $25.9 \pm 5.3$ & $68.8 \pm 31.0$ \\
\hline Normanichthys crockeri & $17.1 \pm 14.4$ & $49.1 \pm 34.9$ & $34.9 \pm 16.9$ & $17.5 \pm 7.4$ & 29,3 & $28.4 \pm 16.3$ & $39.7 \pm 39.2$ & $8.2 \pm 3.9$ \\
\hline Hippoglossina macrops & 2,7 & $5.9 \pm 0.5$ & $14.2 \pm 2.2$ & 3,8 & - & - & $9.2 \pm 11.2$ & $45.7 \pm 27.3$ \\
\hline \multirow[t]{4}{*}{ Sebastes capensis } & $17.8 \pm 6.4$ & $48.8 \pm 32.1$ & $27.2 \pm 14.2$ & $45.9 \pm 65.5$ & $7.5 \pm 6.0$ & 4,8 & $18.2 \pm 8.7$ & 152,8 \\
\hline & \multicolumn{8}{|c|}{ July Slope Station } \\
\hline & \multicolumn{4}{|c|}{ Day } & \multicolumn{4}{|c|}{ Night } \\
\hline & $0-25$ & $25-50$ & $50-100$ & $100-150$ & $0-25$ & $25-50$ & $50-100$ & $100-150$ \\
\hline Hygophum bruuni & $329.4 \pm 195.4$ & $538.5 \pm 643.5$ & $25.9 \pm 17.3$ & $10.5 \pm 2.6$ & 6,1 & $69.2 \pm 19.5$ & $29.8 \pm 18.3$ & - \\
\hline Merluccius gayi & 20,4 & 6,7 & 30,6 & $6.2 \pm 4.6$ & - & - & - & - \\
\hline Engraulis ringens & 25,5 & $7.7 \pm 6.1$ & - & - & - & - & - & - \\
\hline Diogenichthys laternatus & 5,1 & $3.7 \pm 0.5$ & 5,1 & - & - & - & 3,1 & - \\
\hline Sebastes capensis & $15.4 \pm 14.5$ & $3.8 \pm 0.3$ & - & - & 6,1 & - & - & - \\
\hline
\end{tabular}


taxa $(5 \%)$. The most abundant species in both groups were M. gayi (34 larvae $10 \mathrm{~m}^{-2}$ ) and $S$. capensis (22 larvae 10 $\mathrm{m}^{-2}$ ), respectively. Multivariate analysis of the vertical structure of JSH revealed dispersion of larval fishes collected in the shallower depths $(>25 \mathrm{~m})$ and aggregation of ichthyoplankton in middle and deep strata, i.e. between 25 and $75 \mathrm{~m}$ (Fig. 6c, Table 4), regardless of their adult habitat (i.e. S. capensis, N. crockeri and M. gayi, Fig. 8a). No diel structuring of larval fishes was observed during this survey, although some species showed a deepening during night-time (E. ringens, H. macrops and $S$. capensis) while others like larval hake M. gayi were located in the deepest stratum $(50-75 \mathrm{~m})$ during day and night hours (Table 4).

The JSL assemblage showed a reduction in number of taxa captured (26 vs 12), and the overall abundance of all individual taxa except larvae of mesopelagic taxa (Table 2). This group comprised $>90 \%$ of total larvae and was dominated by $H$. bruuni $\left(88 \% ; 148\right.$ larvae $10 \mathrm{~m}^{-}$ $\left.{ }^{2}\right)$. Larvae of demersal species (M. gayi, H. macrops and Genypterus sp.) were the second-most abundant group, contributing 5\% to the total catch. Epipelagic and subtidal/ intertidal taxa were reduced to $\sim 1 \%$ each. Larval fish

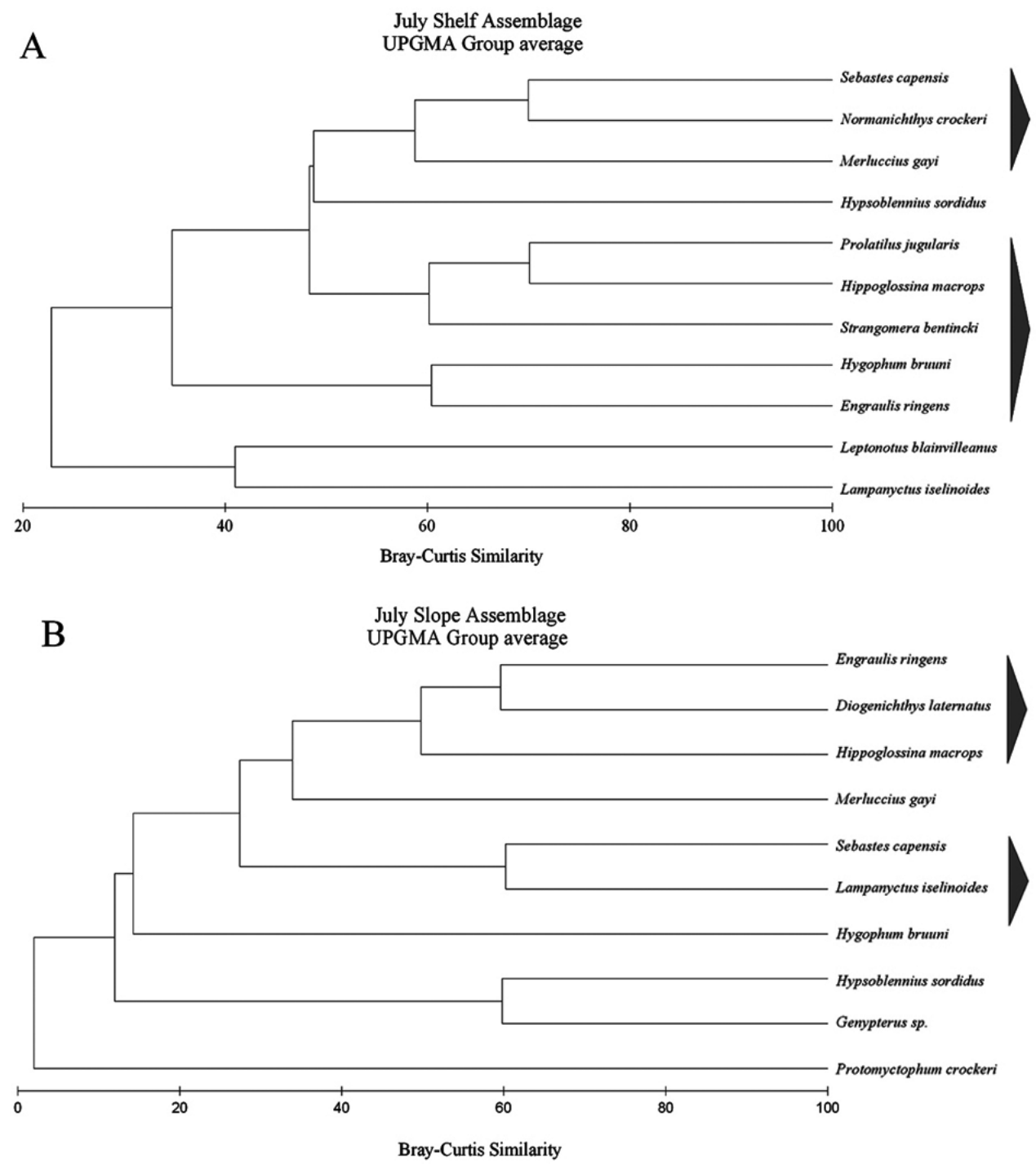

Figure 8

Cluster analysis showing relationship among larval fishes during July 1999 for the a) shelf station and b) slope station

Análisis de dendogramas mostrando la relación entre larvas de peces durante julio de 1999 para la estación de a) plataforma $\mathrm{y}$ b) talud continental 
assemblages in the slope stations also exhibited a depthrelated distribution trend at shallow $(<50 \mathrm{~m})$ and deep $(>50 \mathrm{~m})$ sites (Fig 6d). Most larval fishes were collected in the top $50 \mathrm{~m}$ depth during the day (Table 4). The highly abundant larval stages of $H$. bruuni were collected in higher abundance between 25 and $50 \mathrm{~m}$ of the water column (Table 4). P. crockeri was found only at 50-100 $\mathrm{m}$ depth, and Diogenichthys atlanticus were collected throughout the water column (Fig. 8b).

\section{Discussion}

The interaction between mesoscale physical processes such as upwelling and larval fish assemblages has been well established in several ecosystems of the ocean world. The deep and cold waters upwelled by wind forcing in coastal areas may affect the diversity of larval fishes, by reducing the number of fish species (Olivar et al. 1993, Franco-Gordo et al. 2002), increasing it (Sanchez-Velasco \& Flores-Coto 1994) or displacing larval fishes from slope to shelf areas (Smith \& Suthers 1999).

Plankton and larval fish assemblage spatial variability are influenced by the interaction of topography and currents (Wing et al. 1998, Palma et al. 2006, Muhling \& Beckley 2007). For example, in less than $30 \mathrm{~km}$ crossshelf Smith et al. (1999) described six ichthyoplankton groups associated to coastal, shelf and shelf-break areas of East Australia. Additionally, Marancik et al. (2005) also found distinctive assemblages related to inner, mid and outer continental shelf off the Atlantic coast of United States across a transect of $100 \mathrm{~km}$. Despite neither of both observations correspond to an upwelling system, they are consistent with our results of distinct assemblages for shelf and slope areas. Franco-Gordo et al. (2002) described a spatially homogeneous larval fish assemblage when advective processes dominated the system off the Pacific coast of Mexico. In our case, the studied area has a considerable variability in its topography, with a continental shelf limited by two submarine canyons which directly affect the coastal circulation (Figueroa \& Moffatt 2000, Sobarzo \& Djurfeldt 2004, Sobarzo et al. 2007, Morales et al. 2007) and indirectly may influence the spatial structure of larval fish assemblages off Talcahuano area (see Castillo et al. 1991). In this sense, over the Oregon shelf it has been described that during upwelling season the Astoria Canyon causes currents to flow landward (Hickey 1997), carrying and concentrating normally offshore organisms closer to shore (Bosley et al. 2004, Parnell et al. 2008). In our study, the occurrence of demersal and oceanic larval fishes such as lightfish Maurolicus parvipinnis, hake $M$. gayi, bigeye flounder $H$. macrops and rockfish $S$. capensis over shelf during October may be the result of advective processes associated to upwelling events occurring at shelf break and/or through submarine canyons of Itata and Biobio rivers (transport from shelf break to coastal waters through the subsurface flow that compensates the offshore surface Ekman layer, Smith \& Suthers 1999, Vargas \& Castro 2001, Landaeta \& Castro 2002, Landaeta et al. 2006) and/or by retention near headlands in upwelling shadows during the upwelling relaxation (Wing et al. 1998) or in proximity of frontal areas (Bjorkstedt et al. 2002), rather than spawning events near the coast (Landaeta \& Castro 2006a).

Larval epipelagic fishes were dominated by clupeiform such as anchoveta Engraulis ringens and common sardine Strangomera bentincki. Both species spawns nearshore and associated to rivers during austral winter in the Humboldt ecosystem (Castro et al. 2000, Vargas et al. 2003, Lett et al. 2007, Cubillos et al. 2007) where gelatinous predator levels are low and food items (eggs and nauplii of copepods) are high (Castro et al. 2000). On the other hand, as suggested by Figs. 2 and 3a, offshore advection of surface Ekman layer ( $20 \mathrm{~m}$ depth in the area according to Sobarzo \& Djurfeldt 2004) may partially explain the higher abundance of larval clupeiform fishes in the slope station during October 1998 (austral spring).

Larval fish assemblages were mostly vertically structured irrespective of the time of the day. In northern Chile, Rojas et al. (2002) suggested that larval myctophids (Diogenichthys atlanticus and D. laternatus) and anchoveta $E$. ringens avoided the highly advective upper layer keeping below $80 \mathrm{~m}$ depth. The exception occurred for the OSH, which was structured according to the time of the day. Temporal changes of larval fish assemblages in the shelf station may be triggered by vertical migrations of larval fishes as a way to avoid the offshore surface Ekman layer during the upwelling season (Landaeta \& Castro 2002), and/or by net avoidance of larger larvae.

We found higher abundance and diversity of myctophids larval fishes over shelf and slope stations during July, in association to warmer surface waters moving onshore. This ocurrency pattern is typical throughout the world ocean (Loeb \& Rojas 1988, Nonaka et al. 2000, Sassa et al. 2004). Instead, during October larval myctophids were mostly collected over the shelf break. Although the reproductive cycles of many mesopelagic fishes are weakly seasonal or non-seasonal (Moku et al. 2003), some species (like Lampanyctodes hectoris and Maurolicus spp.) spawns during late winter/ spring at the shelf break (Prosch 1991, Landaeta \& Castro 2002). Along Chile, great abundance of larval Hygophum bruuni, D. atlanticus and Lampanyctus iselinoides are frequently collected in coastal waters during winter time associated to subantarctic waters (Castro et al. 2000, 
Acuña \& Cabrera 2007) and in shallow waters as close to shore as $30 \mathrm{~m}$ (Hernández-Miranda et al. 2003). A potential explanation is that higher diversity of mesopelagic larvae over shelf during July may be consequence of onshore transport of oceanic water parcels, in a similar way to the intrusion of oceanic larval fishes onto the shelf observed by Olivar et al. (1998) in the Agulhas Current.

A similar pattern of abundance and diversity was also evident for intertidal and subtidal larval fishes, being higher during July (austral winter). Intertidal fishes generally have benthic or demersal eggs (Balbontín \& Pérez 1979, Pérez 1981) and its larvae are highly abundant in very nearshore areas (Hernández-Miranda et al. 2003, Miranda Azeiteiro et al. 2006, Borges et al. 2007b). During winter off central Chile there is an increase of the freshwater input and rainfall in the coast (Faundez-Baez et al. 2001), reducing the surface salinity and increasing the extension of river plumes over the shelf (Piñones et al. 2005); by this mechanism intertidal larval fishes may be advected through the inner shelf and may explain the presence of larval blennies (Hypsoblennius sordidus), kyphosids, labrisomids and sciaenids in the shelf station.

Finally, results from this work indicate that the structure of ichthyoplankton assemblages over the shelf and slope in central Chile is influenced by a series of processes acting at different spatial and temporal scales, e.g. an interaction of the spawning behaviour of marine fishes that inhabit the southern part of the Humboldt Current and the frequency of occurrence of mesoscale physical processes such as upwelling events and river runoff. The importance of the oceanographic features in the structure of larval fish assemblages may change seasonally and differs from October (presence of recently upwelled waters) to July (effects of the low salinity layer).

\section{Acknowledgments}

Funding for both cruises was provided by the FONDAPHumboldt Program. During the writing of the manuscript the first author was supported by a CONICYT doctoral scholarship (BECAAT-4040126), R. Veas was supported by a CONICYT doctoral scholarship and FONDECYT grant 1990470 to L.R. Castro. J. Letelier was supported by a CONICYT doctoral scholarship. Thanks to Sociedad de Ciencias del Mar for the Jorge Tomicic scholarship. Finally, we thank to three anonymous referees who helped to improve an early version of this manuscript.

\section{Literature cited}

Acuña E \& MT Cabrera. 2007. Distribución y abundancia de larvas de mictófidos (Pisces: Myctophidae) capturadas durante el crucero biooceanográfico CIMAR 5 Islas
Oceánicas. Ciencia y Tecnología del Mar 30(1): 1-18.

Bakun A. 1996. Patterns in the ocean: ocean processes and marine population dynamics, $323 \mathrm{pp}$. University of California Sea Grant, San Diego \& Centro de Investigaciones Biológicas del Noroeste, La Paz.

Balbontín F \& R Bravo. 1999. Distribución, abundancia y agrupaciones del ictioplancton en un área de desove de merluza frente a Valparaíso. Revista de Biología Marina y Oceanografía 34: 233-260.

Balbontín F \& R Pérez. 1979. Modalidad de postura, huevos y estados larvales de Hypsoblennius sordidus (Bennett) en la Bahía de Valparaíso (Blenniidae: Perciformes). Revista de Biología Marina 16: 311-318.

Berasategui AD, EM Acha \& NC Fernández Araoz. 2004. Spatial patterns of ichthyoplankton assemblages in the Río de la Plata Estuary (Argentina-Uruguay). Estuarine, Coastal and Shelf Science 60: 599-610.

Bjorkstedt EP, LK Rosenfeld, BA Grantham, Y Shkedy \& J Roughgarden. 2002. Distributions of larval rockfishes Sebastes spp. across nearshore fronts in a coastal upwelling region. Marine Ecology Progress Series 242: 215-228.

Borges R, R Beldade \& EJ Gonçalves. 2007a. Vertical structure of very nearshore larval fish assemblages in a temperate rocky coast. Marine Biology 151: 1349-1363.

Borges R, R Ben-Hamadou, MA Chícharo, P Ré \& EJ Gonçalves. 2007b. Horizontal spatial and temporal distribution patterns of nearshore larval fish assemblages at a temperate rocky shore. Estuarine, Coastal and Shelf Science 71: 412-428.

Bosley KL, JW Lavelle, RD Brodeur, WW Wakeeld, RL Emmett, ET Baker \& KM Rehmke. 2004. Biological and physical processes in and around Astoria submarine canyon, Oregon, USA. Journal of Marine Systems 50: 2137.

Castillo G, H Muñoz, H González \& P Bernal. 1991. Daily analysis of abundance and size variability of fish larvae in relation to oceanic water intrusions in coastal areas. Biología Pesquera 20: 21-35.

Castro LR, GR Salinas \& EH Hernández. 2000. Environmental influences on the winter spawning of the anchoveta Engraulis ringens off central Chile. Marine Ecology Progress Series 197: 247-258.

Cox TF \& MAA Cox. 2000. Multidimensional scaling. Monographs on Statistics and Applied Probability 88, 328 pp. Chapman \& Hall/ CRC, Boca Raton.

Cubillos LA, P Ruiz, G Claramunt, S Gacitúa, S Núñez, LR Castro, K Riquelme, C Alarcón, C Oyarzún \& A Sepúlveda. 2007. Spawning, daily egg production and spawning stock biomasa estimation for common sardine (Strangomera bentincki) and anchovy (Engraulis ringens) 
off central southern Chile in 2002. Fisheries Research 86: 228-240.

Daneri G, V Dellarossa, R Quiñones, B Jacob, P Montero \& O Ulloa. 2000. Primary production and community respiration in the Humboldt Current System off Chile and associated oceanic areas. Marine Ecology Progress Series 197: 41-49.

Espinosa-Fuentes ML \& C Flores-Coto. 2004. Cross-shelf and vertical structure of ichthyoplankton assemblages in continental shelf waters of the Southern Gulf of Mexico. Estuarine, Coastal and Shelf Science 59: 333-352.

Fahay MP. 1983. Guide to the early stages of marine fishes occurring in the western North Atlantic Ocean, Cape Hatteras to the southern Scotian Shelf. Journal of the Northwest Atlantic Fishery Science 4: 1-432.

Faundez-Baez P, CE Morales \& D Arcos. 2001. Variabilidad espacial y temporal en la hidrografía invernal del sistema de bahías frente a la VIII región (Chile centro-sur). Revista Chilena de Historia Natural 74: 817-831.

Figueroa D \& C Moffat. 2000. On the influence of topography in the induction of coastal upwelling along the Chilean coast. Geophysical Research Letters 27: 3905-3908.

Franco-Gordo C, E Godínez-Dominguez \& E SuárezMorales. 2002. Larval fish assemblages in waters off central Pacific coast of Mexico. Journal of Plankton Research 24: 775-784.

Gray CA \& MJ Kingsford. 2003. Variability in thermocline depth and strength, and relationships with vertical distributions of fish larvae and mesozooplankton in dynamic coastal waters. Marine Ecology Progress Series 247: 211224.

Grunewald AC, CE Morales, HE González, C Sylvester \& LR Castro. 2002. Grazing impact of copepod assemblages and gravitacional flux in coastal and oceanic waters off central Chile during two contrasting seasons. Journal of Plankton Research 24: 55-67.

Hernández-Miranda E, AT Palma \& FP Ojeda. 2003. Larval fish assemblages in nearshore coastal waters off central Chile: temporal and spatial patterns. Estuarine Coastal and Shelf Science 56: 1075-1092.

Hickey BM. 1997. The response of a steep-sided, narrow canyon to time-variable wind forcing. Journal of Physical Oceanography 27: 697-726.

Landaeta MF \& LR Castro. 2002. Spring spawning and early nursery zone of the mesopelagic fish Maurolicus parvipinnis at the coastal upwelling zone off Talcahuano, central Chile. Marine Ecology Progress Series 226: 179191.

Landaeta MF \& LR Castro. 2006a. Spawning and larval survival of the Chilean hake Merluccius gayi under later summer upwelling conditions in the Gulf of Arauco, central Chile. Fisheries Research 77: 115-121.

Landaeta MF \& LR Castro. 2006b. Larval distribution and growth of the rockfish, Sebastes capensis (Sebastidae, Pisces), in the fjords of southern Chile. ICES Journal of Marine Science 63: 714-724.

Landaeta MF, GA Herrera, M Pedraza, CA Bustos \& LR Castro. 2006. Reproductive tactics and larval development of bigeye flounder, Hippoglossina macrops, off central Chile. Journal of the Marine Biological Association of the United Kingdom 86: 1253-1264.

Lett C, P Penven, P Ayón \& P Freón. 2007. Enrichment, concentration and retention processes in relation to anchovy (Engraulis ringens) eggs and larvae distributions in the northern Humboldt upwelling ecosystem. Journal of Marine Systems 64: 189-200.

Loeb VJ \& O Rojas. 1988. Interannual variation of ichthyoplankton composition and abundance relations off northern Chile 1964-1983. Fishery Bulletin 86: 1-24.

Marancik KE, LM Clough \& JA Hare. 2005. Cross-shelf and seasonal variation in larval sh assemblages on the southeast United States continental shelf off the coast of Georgia. Fishery Bulletin 103: 108-129.

Miranda Azeiteiro U, L Bacelar-Nicolau, P Resende, F Gonçalves \& MJ Pereira. 2006. Larval fish distribution in shallow coastal waters off North Western Iberia (NE Atlantic). Estuarine, Coastal and Shelf Science 69: 554566.

Moku M, A Tsuda \& K Kawaguchi. 2003. Spawning season and migration of the myctophid fish Diaphus theta in the Western North Pacific. Ichthyological Research 50(1): 5258.

Montero P, G Daneri, LA Cuevas, HE González, B Jacob, L Lizárraga \& E Menschel. 2007. Productivity cycles in the coastal upwelling area off Concepción: The importance of diatoms and bacterioplankton in the organic carbon flux. Progress in Oceanography 75: 518-530.

Morales CE, HE González, SE Hormazábal, G Yuras, J Letelier \& LR Castro. 2007. The distribution of chlorophyll- $a$ and dominant planktonic components in the coastal transition zone off Concepción, central Chile, during different oceanographic conditions. Progress in Oceanography 75: 452-469.

Moser HG. 1996. The early stages of fishes in the California Current region. California Cooperative Oceanic Fisheries Investigations.Atlas 33: 1-1505.

Muhling BA \& LE Beckley. 2007. Seasonal variation in horizontal and vertical structure of larval fish assemblages off south-western Australia, with implications for larval transport. Journal of Plankton Research 29(11): 967-983. 
Neira FJ, AG Miskiewicz \& T Trnski. 1998. Larvae of Temperate Australian Fishes. Laboratory guide for larval fish identification, $474 \mathrm{pp}$. University of Western Australia Press, Nedlands.

Nonaka RH, Y Matsuura \& K Suzuki. 2000. Seasonal variation in larval fish assemblages in relation to oceanographic conditions in the Abrolhos Bank region off eastern Brazil. Fishery Bulletin 98: 767-784.

Okubo A. 1980. Diffusion and ecological problems: mathematical models, 254 pp. Springer-Verlag, Berlin.

Olivar MP. 1990. Spatial patterns of ichthyoplankton distribution in relation to hydrographic features in the Northern Benguela region. Marine Biology 106: 39-48.

Olivar MP \& PA Shelton. 1993. Larval fish assemblages of the Benguela Current. Bulletin of Marine Science 53(2): 450-474.

Olivar MP, J Salat \& LE Beckley. 1998. Evidence of larval fish displacement associated with surface water movements. South African Journal of Marine Science 19: 233-244.

Palma AT, LM Pardo, R Veas, C Cartes, M Silva, K Manríquez, A Díaz, C Muñoz \& FP Ojeda. 2006. Coastal brachyuran decapods: settlement and recruitment under contrasting coastal geometry conditions. Marine Ecology Progress Series 316: 139-153.

Parnel MM, RL Emmett \& RD Brodeur. 2008. Ichthyoplankton community in the Columbia River plume off Oregon: effects of fluctuating oceanographic conditions. Fishery Bulletin 106: 161-173.

Peguero-Icaza M, L Sánchez-Velasco, MF Lavín \& SG Marinone. 2008. Larval fish assemblages, environment and circulation in a semienclosed sea (Gulf of California, Mexico). Estuarine, Coastal and Shelf Science 79: 277288.

Pérez R. 1981. Desarrollo embrionario y larval de los pejesapos Syciases sanguineus y Gobiesox marmoratus en la Bahía de Valparaíso, Chile, con notas sobre su reproducción (Gobiesocidae: Pisces). Investigaciones Marinas 9: 1-23.

Piñones A, A Valle-Levinson, DA Narváez, CA Vargas, SA Navarrete, G Yuras \& JC Castilla. 2005. Wind-induced diurnal variability in river pluma motion. Estuarine, Coastal and Shelf Science 65(3): 513-525.

Prosch RM. 1991. Reproductive biology and spawning of the myctophid Lampanyctodes hectoris and the sternoptychid Maurolicus muelleri in the southern Benguela ecosystem. South African Journal of Marine Science 10(1): 241-252.

Rodriguez-Graña L \& LR Castro. 2003. Ichthyoplankton distribution off the Península de Mejillones, Chile $\left(23^{\circ} \mathrm{S}\right.$, $71^{\circ} \mathrm{W}$ ), under variable hydrographic conditions during the austral summer and winter of the 1997 El Niño. Hydrobiologia 501: 59-73.
Rojas PM, R Escribano \& VH Marín. 2002. Fish larvae distribution off Mejillones Peninsula (northern Chile) during a coastal upwelling event in spring 1999: interactions with the cold upwelling plume. Fisheries Oceanography 11(4): 233-244.

Sabatés A. 2004. Diel vertical distribution of fish larvae during the winter-mixing period in the Northwestern Mediterranean. ICES Journal of Marine Science 61: 12431252.

Sanchez-Velasco L \& C Flores-Coto. 1994. Larval fish assemblages at the Yucatan shelf and in the Mexican Caribbean Sea during the upwelling period (Spring, 1985). Scientia Marina 58(4): 289-297.

Sanchez-Velasco L, E Beier, C Avalos-García \& MF Lavín. 2006. Larval fish assemblages and geostrophic circulation in Bahía de la Paz and the surrounding southwestern region of the Gulf of California. Journal of Plankton Research 28(11): 1081-1098.

Sanchez-Velasco L, SPA Jiménez-Rosenberg \& MF Lavín. 2007. Vertical distribution of fish larvae and its relation to water column structure in the southwestern Gulf of California. Pacific Science 61(4): 533-548.

Sassa C, K Kawaguchi, Y Hirota \& M Ishida. 2004. Distribution patterns of larval myctophid fish assemblages in the subtropical-tropical waters of the western North Pacific. Fisheries Oceanography 13(4): 267-282.

Smith KA \& IM Suthers. 1999. Displacement of diverse ichthyoplankton assemblages by a coastal upwelling event on the Sydney shelf. Marine Ecology Progress Series 176: 49-62.

Smith KA, MT Gibbs, JH Middleton \& IM Suthers. 1999. Short term variability in larval assemblages of the Sydney shelf: tracers of hydrographic variability. Marine Ecology Progress Series 178: 1-15.

Sobarzo M \& L Djurfeldt. 2004. Coastal upwelling process on a continental shelf limited by submarine canyons, Concepción, central Chile. Journal of Geophysical Research 109(C12): C12012.1-C12012.20

Sobarzo M, L Bravo, D Donoso, J Garcés-Vargas \& W Schneider. 2007. Coastal upwelling and seasonal cycles that influence the water column over the continental shelf off central Chile. Progress in Oceanography 75: 363-382.

Sponaugle S, RK Cowen, A Shanks, SG Morgan, JM Leis, J Pineda, GW Boehlert, MJ Kingsford, KC Lindeman, C Grimes \& JL Munro. 2002. Predicting self-recruitment in marine populations: biophysical correlates and mechanisms. Bulletin Marine Science 70(Suppl): 341-375.

Strub PT, JM Mesias, V Montecino, J Rutlland \& S Salinas. 1998. Coastal ocean circulation off western South America. 
In: Robinson AR \& KH Brink (eds), The Sea vol. 11: 273 313. John Wiley \& Sons, New York.

Vargas CA \& LR Castro. 2001. Spawning of Chilean hake (Merluccius gayi) in the upwelling system off Talcahuano in relation to oceanographic features. Scientia Marina 65(2): 101-110.

Vargas CA, S Araneda \& G Valenzuela. 2003. Influence of tidal phase and circulation of larval fish distribution in a partially mixed estuary, Corral Bay, Chile. Journal of the Marine Biological Association of the United Kingdom 83(1): 217-222.
Vélez JA, W Watson, W Arntz, M Wolff \& SB SchnackSchiel. 2005. Larval fish assemblages in Independencia Bay, Pisco, Peru: temporal and spatial relationships. Marine Biology 147: 77-91.

Wing SR, LW Bostford, SL Ralston \& JL Largier. 1998. Meroplanktonic distribution and circulation in a coastal retention zone of the northern California upwelling system. Limnology and Oceanography 43(7): 1710-1721.

Recibido el 2 de julio de 2008 y aceptado el 30 de septiembre de 2008 